an easy-to-read leaflet for carers detailing local support services.

This has also been enhanced through joint social media activity, with a Twitter and Facebook account created along with the local \#KirkleesThinkCarers.

Future plans include further collaboration on carers' issues, exploring service development and integration, development of new resources and through the medium of a shared social media account, keeping carers updated about the services that we all offer and a central point of contact.

\section{P-194 IMPROVING CARERS' SUPPORT SERVICES AT MARIE CURIE HOSPICE, NEWCASTLE}

Emily Buck. Marie Curie, Newcastle, UK

\subsection{6/bmispcare-2019-HUKNC.216}

Carers have their own concerns, anxieties and stresses, and the complexities of these can influence their ability to fulfil this role (Trower \& Reed, 2016). In turn this can significantly impact upon the patients' symptoms and psychological state, and has the potential to prevent patients from being cared for and dying in the place of their choosing (Janze \& Henriksson, 2014). In claiming to provide a holistic service for palliative patients it is therefore vital that carers' needs be assessed and addressed.

With this philosophy in mind, in the financial year 2018/ 19 the Marie Curie Hospice (Newcastle) engaged with the Commissioning for Quality and Innovation (CQUIN) with the aim of improving the support offered to carers of our patients.

A multi-disciplinary steering group was initially formed and priorities for service improvements were developed.

The formal assessment of carers needs was felt to be paramount and we therefore embarked upon the hospice-wide introduction of the Carers' Support Needs Assessment Tool (CSNAT), (Ewing \& Grande, 2013). Through engagement with the multidisciplinary team, support services have been established or developed which include mindfulness and art therapy groups, 1:1 relaxation/hypnotherapy and expansion of bereavement services to include a 'social' group and a Sons, Daughters and Siblings group. We are using information technology systems to aid the timely identification of carers and have introduced specific information resources in the form of a carers' hub and a carers' specialist section in the patients' bedside folder pack.

Many of the initiatives are in their infancy and so their impact has not yet been evaluated, although much positive feedback has been received. Moving forward there are plans to audit the use of CSNAT and research the impact of these service developments.

\section{P-195 COLLABORATIVE WORKING BETWEEN CHILDREN AND ADULT PALLIATIVE CARE/HOSPICE SERVICES}

${ }^{1}$ Louise Smith, ${ }^{2}$ Emma Longford, ${ }^{1}$ Andy Curtis. ${ }^{1}$ Claire House Children's Hospice, Wirral, UK; Wirral Hospice St John's, Wirral, UK

10.1136/bmjspcare-2019-HUKNC.217

Background It is well documented that transition is a scary, isolating process for young persons (YPs) and their families, with clear gaps in services (Care Quality Commission, 2014). Claire House collaborated with Wirral Hospice for an individual patient and positive outcomes were reported. We aim to replicate this offer to all YPs, offering a coordinated, continuous collaboration and opportunity to build relationships safely.

Aim To provide a collaborative service for all YPs, comprising a five-year overlap of services. To manage palliative care needs jointly, improve symptom management and quality of life. This collaboration will provide opportunity for adult services to extend knowledge of previously childhood conditions (Care Quality Commission, 2014).

Method Families were invited to joint presentation evenings facilitating early relationship building and allowing exploration of what is wanted (NICE, 2016; Chambers, 2015). Agreed to offer joint clinics (Chambers, 2015), parallel planning discussions, symptom management, and social events to enable YPs to build relationships.

Expected results Increased quality of life for YPs, improved symptom management, fewer hospital admissions, more support for families (both peer and professional), greater opportunity to build sustainable relations. The team will seek purposeful feedback at regular YP sessions, and professional feedback.

Conclusions This project will provide a choice of services offering support to YPs and their families throughout transition and providing a continuous, collaborative relationship with SPC services. It will enable adult services to gain the right skills to meet the complex needs of increasingly dependent YPs.

Innovative/of interest Children are living longer, increasing the need for access to adult palliative care. There exists a gap in knowledge of adult services, for previously exclusive childhood diseases and inequitable service provision. This project will enable children's hospices and adult palliative care services to work together and improve the struggle that families and YPs face with transition and relationship-building with adult services.

\section{P-196 OUR SPACE - A PLACE TO JUST BE...}

Elaine Pugh. Woodlands Hospice, Liverpool, UK

\subsection{6/bmjspcare-2019-HUKNC.218}

Background 'Our Space' is an exciting new development created in partnership with children and young people, we recognise the rights and needs of children to grieve and be heard. Most of the children and young people we support are bereaved or have a family member who is being cared for by the hospice team. We were determined to advocate and work in partnership with children and young people to provide child-centred compassionate support, to increase opportunities to express feelings of grief and loss, to create an environment that lends itself to children and young people - a dedicated space.

How we did this The Family Support team carried out a number of consultations working with services users and agencies from across the hospice community.

The findings Children and young people reported a lack of understanding from a wide range of professionals. Not being respected for their knowledge, experience of living with a dying parent or relative. 
Often excluded from discussion of care regarding a parent for whom they were main carer or involved in caring. Not always given the opportunity to talk about the impact of supporting and living with a dying parent or relative.

Children and young people shared heartfelt personal experiences which supported our concerns regarding the following:

Young carers described the challenges accessing support, spoke candidly about the restrictions and barriers they faced, and talked about the need to have some time away, to work out their feelings and wanting a safe space.

What we did A young persons' advisory group was formed and led on the development and design of 'Our Space'.

Collaboration with the local community to fundraise for the build of 'Our Space' in the hospice's gardens.

Opening of a dedicated resource for children and young people within an adult hospice ensuring their voices, views hopes and aspirations are at the heart of the care and support we provide.

Development of peer support groups.

\section{P-197 EXPLORING THE SUCCESS OF PALLIATIVE CARE PROVISION IN UGANDA}

Maya Connolly. Wirral University Teaching Hospital, Merseyside, UK

\subsection{6/bmjspcare-2019-HUKNC.219}

Background The majority of palliative care needs (78\%) are in low to middle-income countries; despite this, palliative care provision remains poor. Over the past 20 years, developing palliative services has increasingly become a priority for international organisations. Uganda has developed the most effective palliative care service in Sub-Saharan Africa. Globally, its palliative care services have been classed (alongside 20 other countries) as one of the most advanced palliative care services, in which 'hospice-palliative care services are at a stage of advanced integration into mainstream service provision'.

Aims Explore the potential factors which aided the development of palliative care services in Uganda and consider the lessons we can learn from this successful case study.

Methods Multi-source literature review.

Results Hospice Africa Uganda (HAU) was established in Uganda (1993) by a UK based palliative care physician. The hospice aimed to create a model of palliative care which was affordable, culturally acceptable and could be adapted to other countries in Africa.

Key factors this poster will explore, include:

- Ensured prescribing and giving opiates was not illegal and that there was sufficient supply at a reasonable cost to the service - this remains a barrier to the delivery of palliative care internationally;

- Strong research focus - service evaluation and improvement;

- Healthcare model - Integrated Community Home-Based Care which trained community volunteers supported and supervised by clinicians - care is delivered at a combination of hospitals, hospices and in the community;

- Integration of palliative care education into undergraduate and postgraduate programmes - this has led to a shift in attitudes towards palliative care and in the understanding of the importance of respect and individualised care;

- The social and political climate - Uganda was rebuilding itself with significant international financial support; the civil war had come to an end seven years before.

\section{P-198 NURSE-LED CARE FOR NON-COMPLEX DYING: A COLLABORATION BETWEEN HOSPICE AND ACUTE HOSPITAL}

Carly Wills, Sarah Grove. Arthur Rank Hospice Charity, Cambridge, UK

10.1136/bmjspcare-2019-HUKNC.220

The acute trust has severe bed capacity issues. Patients were dying on busy, acute hospital wards but often did not meet the criteria for admission to a specialist hospice bed due to lack of specialist palliative care needs. We had a new purposebuilt hospice with extra non-commissioned beds that we knew could benefit our community.

Our intention was to allow people to have a dignified death in more suitable surroundings. This resulted in us launching the nurse-led bed initiative; skilled nurses who are trained in caring for people at end of life run the beds with minimal medical input.

The service in its first year has seen over 250 patients, saving the acute trust over 2600 bed days. The average length of stay for patients is 10 days. The shortest stay was one hour; the longest 87 days. The feedback from patients and families shows it has been an overwhelming success. Nurses feel empowered to make decisions regarding patient care and have been working in partnership with teams at Cambridge University Hospital to champion the pilot and communicate its success. Patients are feeling more in control by having an alternative to staying in hospital. Families are not limited by visiting hours or other restrictions and can stay overnight. All rooms have access to outside and pets can visit.

The next step is to make it a seven day a week service as, due to current resources, it runs Monday to Friday. This may increase occupancy in the beds, thus meaning that an even greater number of patients are likely to have a better experience at the end of life.

'It's just everything it should be; peaceful, light, airy, clean and staff that are attentive and know what's going on. I press the buzzer and they are there.' Patient's relative.

\section{P-199 UK HOSPICE HEALTH LINKS WITH INTERNATIONAL PALLIATIVE CARE PROVIDERS: BENEFITS AND CHALLENGES}

Jan Eversfield. Royal Free Hospital NHS Trust, London, UK

10.1136/bmjspcare-2019-HUKNC.221

United Kingdom (UK) hospices historically have a history of health links or partnerships with resource-constrained countries to share learning through 'twinning' programmes, the donation of equipment or supplies. With no central database of these links, this study sought to map current, active health links with UK hospices.

Every UK hospice was contacted and there was a $22 \%$ response rate. The author conducted semi-structured telephone interviews with all of the respondents to understand their active health link, the benefits and challenges of the health links and interviews offered advice to UK hospices looking to develop health links in the future. The majority of the health links were educational in focus. Ten hospices had active health links with resource-constrained countries, while a further five had developed international health links with countries not 